\title{
Enfermedad de Still del adulto asociado a compromiso pulmonar
}

\author{
Andrés Felipe Posada López¹, Viviana Yepes Gaviria², Hernán Darío Aguirre Henao³, \\ María Luisa Quevedo Cámera ${ }^{4}$ \\ ${ }^{1,3}$ Médico Residente de Medicina Interna Universidad de la Sabana, Bogotá, Colombia. ${ }^{2}$ Médico General Fundación Universitaria San Martín, \\ Medellín, Colombia. ${ }^{4}$ Estudiante de Medicina. Universidad de la Sabana. Hospital Universitario de la Samaritana, Bogotá D. C., Colombia.
}

\section{Resumen}

La enfermedad de Still del adulto es una patología sistémica poco frecuente, de etiología aún no clara que afecta principalmente a adultos jóvenes y que se caracteriza por presentar fiebre, rash evanescente color rosa salmón, odinofagia, artralgia 0 artritis y en algunos casos hepatoesplenomegalia y adenomegalias. En el siguiente artículo presentamos el caso de una paciente de 21 años que presentó dichas manifestaciones asociadas a compromiso pulmonar, elevación de transaminasas y ferritina, leucocitosis con anticuerpos anticitoplasma de neutrófilos (ANCAS) y anticuerpos antinucleares (ANAs) positivos que hacen dudar del diagnóstico de la enfermedad.

\section{Abstract}

Still's disease is an uncommon systematic disorder of unknown etiology which affects young adults characterized by high spiking fever, evanescent salmon color rash, odynophagia, arthritis and in some cases hepatosplenomegaly and lymphadenophaties. The next article will present the case of a 21 year old female which presented these manifestations associated with pulmonary involvement, elevated transaminases and ferritin, marked leukocytosis with ANA's and ANCA's positive that makes this case doubtful for Still's disease.

\section{Caso clínico}

Paciente femenina de 21 años con cuadro clínico de 4 meses de evolución consistente en aparición de lesiones en piel maculares, eritematosas y evanescentes localizadas en tronco y extremidades, con predominio en miembros superiores, asociadas a fiebre de $39^{\circ} \mathrm{C}$ y odinofagia. Consultó en múltiples ocasiones en las cuales se manejó como faringoamigdalitis bacteriana aguda sin obtenerse mejoría con el manejo antibiótico con Fenoximetilpenicilina. Persiste con los síntomas previamente descritos y aparición de artralgias y sinovitis de pequeñas y grandes articulaciones (metacarpofalángicas proximales, interfalángicas proximales, codos y rodillas bilaterales) no migratoria, de componente simétrico con limitación para la movilización, sin presencia de rigidez matinal; también se documentan adenomegalias cervicales y esplenomegalia, fiebre vesperal con concurrencia de rash evanescente de color rosa salmón (Figura 1) y reporte de ferritina sérica de $31.120 \mathrm{ng} / \mathrm{ml}$, leucocitosis, ANAs positivo (1/80 patrón homogéneo), anti-DNA negativo, ANCAS por inmunofluorescencia indirecta (IFI) positivos pero con Antimieloperoxidasa (MPO) y An-

\section{Correspondencia}

Andrés Felipe Posada López; Dirección Carrera 55 No. 160-63, Apto 1101, Torre C Bogotá, Colombia.

E-mail: andrespolo2@hotmail.com 

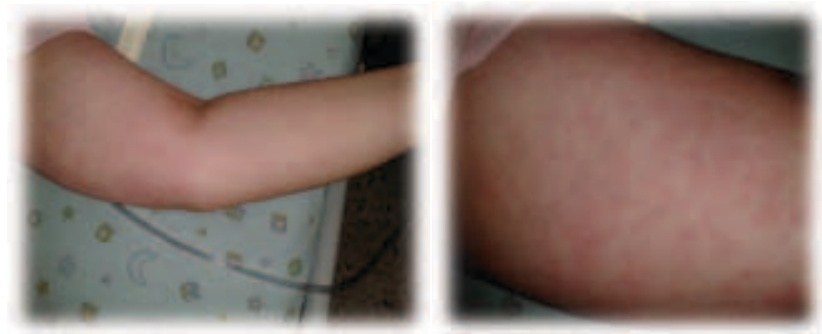

Figura 1. Rash evanescente de color salmón presentado por la paciente.

\begin{tabular}{|c|c|c|c|}
\hline Glicemia (mg/dl) & 86 & PCR (mg/dl) & 177,3 \\
\hline Na (mEq/L) & 144 & Leucocitos & 12.300 \\
\hline K (mmol/l) & 4,6 & PNM & $55 \%$ \\
\hline CL (mmol/l) & 103 & Linfocitos & $45 \%$ \\
\hline Albúmina & 3,44 & Hb & 15,3 \\
\hline VSG (mg/dl) & 45 & HTO & 43 \\
\hline BUN (mg/dl) & 10,8 & Plaquetas & 166.000 \\
\hline Creatinina (mg/dl) & 0,76 & Hemocultivos & Negativos \\
\hline
\end{tabular}

Tabla 1. Glicemia, electrolitos, albúmina, VSG, azoados, PCR, cuadro hemático.

\begin{tabular}{|c|c|c|c|c|c|}
\hline AST (mg/dl) & 171 & VIH & Negativo & ANA's & Positivo \\
\hline ALT (mg/dl) & 139 & VDRL & No reactivo & ANTI(LA) & $1,3(-)$ \\
\hline $\begin{array}{c}\text { Bilirrubina Total } \\
\text { (mg/dl) }\end{array}$ & 0,3 & AGs Hep B & $0,41(-)$ & ANTI(R0) & $2,1(-)$ \\
\hline $\begin{array}{c}\text { Bilirrubina } \\
\text { Directa (mg/dl) }\end{array}$ & 0,12 & $\begin{array}{c}\text { Ferritina } \\
\text { (ng/ml) }\end{array}$ & 31.120 & $\begin{array}{c}\text { Complemento } \\
\text { Sérico C3 }\end{array}$ & 102,7 \\
\hline $\begin{array}{c}\text { Fosfatasa } \\
\text { Alcalina (mg/dl) }\end{array}$ & 172 & ANCA's & Positivo & $\begin{array}{c}\text { Complemento } \\
\text { Sérico C4 }\end{array}$ & 20,00 \\
\hline $\begin{array}{c}\text { Procalcitonina } \\
\text { (ng/ml) }\end{array}$ & $<0,5$ & MP0 & $2,9(-)$ & Anti - P3 & $2,41(-)$ \\
\hline
\end{tabular}

Tabla 2. Función hepática, VIH, VDRL, Antígenos HepB, ferritina, ANCA's, ANA's, ANTI(LA), ANTI(RO).

tiproteinasa 3 (Anti-P3) Negativos, anticitrulinas (CCP) negativo y biopsia de ganglio cervical que sugiere reactividad (Tablas 1 y 2 ).

Se conceptúa que la paciente cumple con criterios de Yamaguchi para enfermedad de Still del adulto (Tabla 3), por lo que se inició manejo con antiinflamatorios no esteroideos (AINES) sin evidenciarse mejoría clínica a los 15 días de iniciada la terapia, considerándose adición de esteroide oral (Prednisolona) a dosis de $1 \mathrm{mg} / \mathrm{kg} / \mathrm{día} \mathrm{du}-$ rante 1 mes; sin embargo, llama la atención la aparición de sintomatología respiratoria de 3 semanas de evolución dada por tos con expectoración no purulenta asociada a disnea clasificada por escala modificada del Medical Research Council (MRCm) 3/4.
Por lo anteriormente mencionado se realiza radiografía de tórax que evidenció opacidades de ocupación alveolar bilaterales que sugieren proceso infeccioso multilobar y se procede a solicitar baciloscopias, con resultado negativo para Mycobacterium tuberculosis al igual que el gram y cultivo de esputo para gérmenes comunes. Igualmente se practica tomografía axial computarizada de tórax de alta resolución (TACAR), la cual demuestra infiltrados bilaterales reticulonodulares con opacidades de ocupación alveolar que sugieren tuberculosis pulmonar como primera posibilidad (Figura 2); por este motivo se lleva a cabo lavado bronquioalveolar, el cual evidencia predominio linfocitario, con cultivo y reacción en cadena de polimerasa (PCR) en tiempo real negativos para Mycobacterium tuberculosis, sin evidencia de hemosiderófagos, ni descenso de hemoglobina sérica que sugieran compromiso por hemorragia alveolar. Para este momento, se considera importante descartar vasculitis de pequeño vaso (Poliangiitis con granulomatosis) $\mathrm{u}$ otra vasculitis por antecedente de infección del tracto respiratorio superior crónica y por hallazgos de infiltrados pulmonares asociado a ANCAS positivos pero mieloperoxidasa (MPO) y antiproteinasa (P3) negativos, considerándose como epifenómeno asociado a la enfermedad de Still.

Se considera entonces que se trata de compromiso pulmonar secundario a enfermedad de Still del adulto (Neumonitis intersticial) y se inició manejo inmunosupresor con Metotrexate dosis de $15 \mathrm{mg} / \mathrm{semanal}$ durante

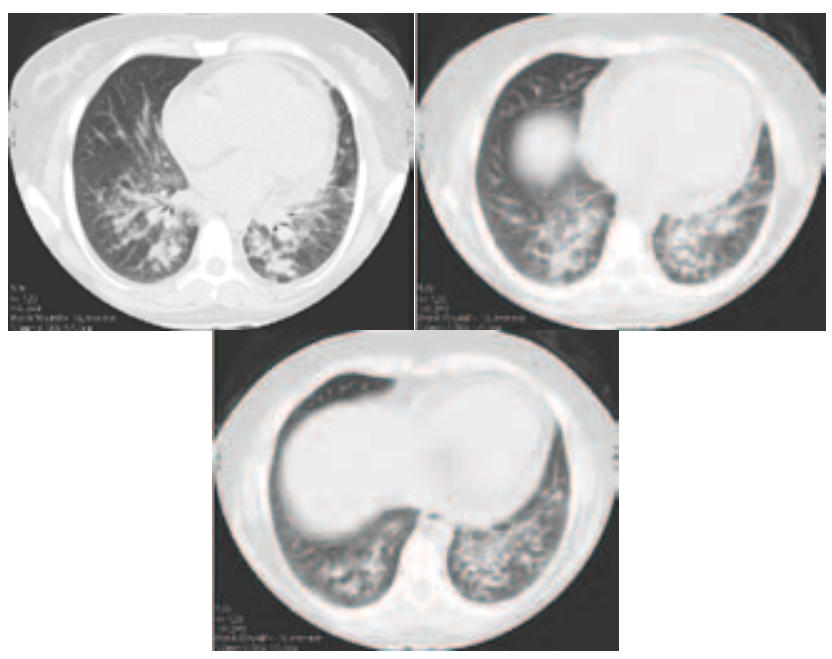

Figura 2. Tomografía axial computarizada de tórax con opacidades de ocupación alveolar y compromiso reticulonodular apical y basal bilateral. 

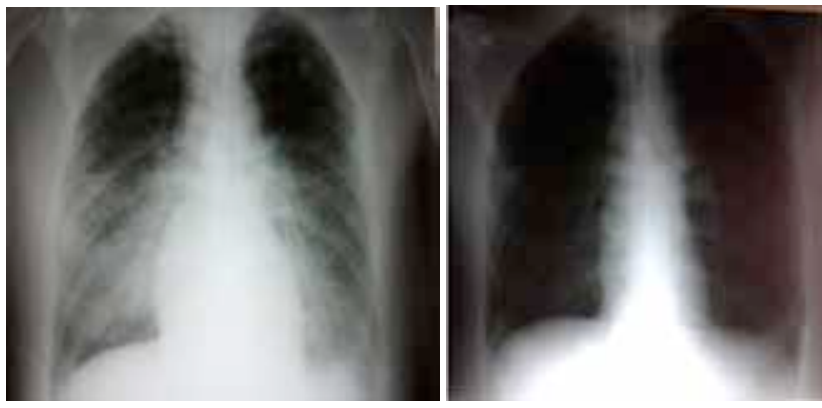

Figura 3. Mejoría de las opacidades de ocupación alveolar posterior al inicio del tratamiento inmunosupresor con Tocilizumab.

1 mes, sin obtenerse adecuada respuesta clínica, con persistencia de sintomatología, compromiso pulmonar y actividad inflamatoria evidenciada en cuadro hemático (Leucocitosis) y valor elevado de la Proteína C Reactiva.

Se decide finalmente inicio de terapia con inhibidor de la interleuquina 6 (Tocilizumab), con mejoría significativa del compromiso pulmonar, evidenciando desaparición de las opacidades de ocupación alveolar en la radiografía de control a los 20 días de iniciada dicha terapia y con descenso de los marcadores inflamatorios y mejoría de las manifestaciones sistémicas (Figura 3).

\section{Discusión}

La enfermedad de Still del adulto es una patología inflamatoria sistémica de etiología desconocida y poco frecuente, que afecta adultos jóvenes. El diagnóstico se hace después de la exclusión de infecciones, tumores malignos y otras enfermedades autoinmunes, utilizando los criterios de Yamaguchi ${ }^{4}$.

Se considera que casi todos los pacientes tienen un incremento de la sedimentación globular, proteína C reactiva, amiloide sérico, haptoglobina, ferritina e hipercomplementemia. La elevación de la ferritina es clave

\begin{tabular}{|c|c|c|}
\hline & Criterios de Cush & Criterios de Yamaguchi \\
\hline \multirow{3}{*}{ Criterios } & - Fiebre sobre $39^{\circ} \mathrm{C}$ & - Fiebre sobre $39^{\circ} \mathrm{C}>1$ semana \\
Mayores & - Artralgias/artritis & - Artralgias/artritis $>2$ semanas \\
& - Factor reumatoide $<1: 80$ & - Rash evanescente \\
& - Ac antinucleares & - Leucocitosis $>10.000$ \\
& - Neutrófilos $>80 \%$ \\
\hline & - Leucocitosis $>15.000 / \mathrm{mm}^{3}$ & - Odinofagia \\
Criterios & - Rash evanescente & - Adenomegalias \\
Menores & - Pleuritis o pericarditis & - Aumento enzimas hepáticas \\
& - Hepatomegalia - esplenomegalia & - F. Reumatoideo $(-)$ \\
& - Adenomegalias & - Ac antinucleares $(-)$ \\
\hline \multirow{2}{*}{ Diagnóstico } & -3 mayores & -5 criterios \\
& -2 menores & -2 deben ser mayores \\
\hline
\end{tabular}

Tabla 3. Criterios diagnósticos para enfermedad de Still del adulto. para el diagnóstico de la enfermedad de Still del adulto $y$, aunque es poco específica, los niveles mayores de 1000 $\mathrm{ng} / \mathrm{ml}$ o más de cinco veces su valor normal sugiere el diagnóstico con una sensibilidad del $80 \%$ y una especificidad del $46 \%$, siendo más confiables los valores de Ferritina Glicosilada $\leq 20 \%{ }^{7}$. La positividad de los ANCAS por medio de IFI puede ser considerada como un epifenómeno más que una asociación con vasculitis ${ }^{8}$.

Dentro de las manifestaciones poco comunes encontramos el compromiso pulmonar, con una frecuencia entre el 5 y el 25\% de los casos, siendo más prevalente la neumonitis con infiltrado alveolar e intersticial bilateral que puede ser transitorio, pero en algunos casos puede complicarse hasta generar síndrome de dificultad respiratoria aguda'. Alrededor de un tercio de los pacientes $(31,5 \%)$ con compromiso pulmonar tienen derrame pleural con exudado linfocitario ${ }^{10}$. La pleuritis unilateral o bilateral se observa en el $12-53 \%$ y es muchas veces vista poco después de la aparición de la enfermedad o durante una exacerbación de ésta ${ }^{11}$.

La patogenia del compromiso pulmonar es desconocida; los infiltrados intersticiales podrían estar relacionados con la infiltración de los linfocitos $\mathrm{T}$ y citoquinas del perfil TH1 durante la enfermedad de Still ${ }^{12}$. Los niveles elevados de IL-18 en el suero y tejido pulmonar se han visto durante las fases agudas de la enfermedad y están asociados con niveles elevados de ferritina en suero ${ }^{13}$.

La enfermedad pulmonar intersticial se caracteriza por infiltrados transitorios en la radiografía, generalmente asintomáticos y puede verse hasta en 13\% de los casos. La espirometría revela un defecto pulmonar restrictivo con baja difusión de $\mathrm{CO}_{2}$. La biopsia transbronquial incluye fibrosis intersticial no específica, alveolitis aguda con paredes alveolares engrosadas, hiperplasia de neumocitos tipo II, formación de la membrana hialina e infiltración linfocítica ${ }^{14}$. Estos hallazgos resuelven bien bajo el tratamiento con antiinflamatorios no esteroideos, corticoesteroides o manejo inmunosupresor ${ }^{15}$.

La neumonía intersticial en enfermedad de Still es aguda y se detecta como opacidades alveolares transitorias, como fue visto en el caso descrito ${ }^{16}$. La neumonía criptogénica organizada ha sido descrita en varias enfermedades autoinmunes: artritis reumatoidea, lupus eritematoso sistémico, polimiositis/dermatomiositis y esclerodermia; sin embargo, la asociación de Neumonía 


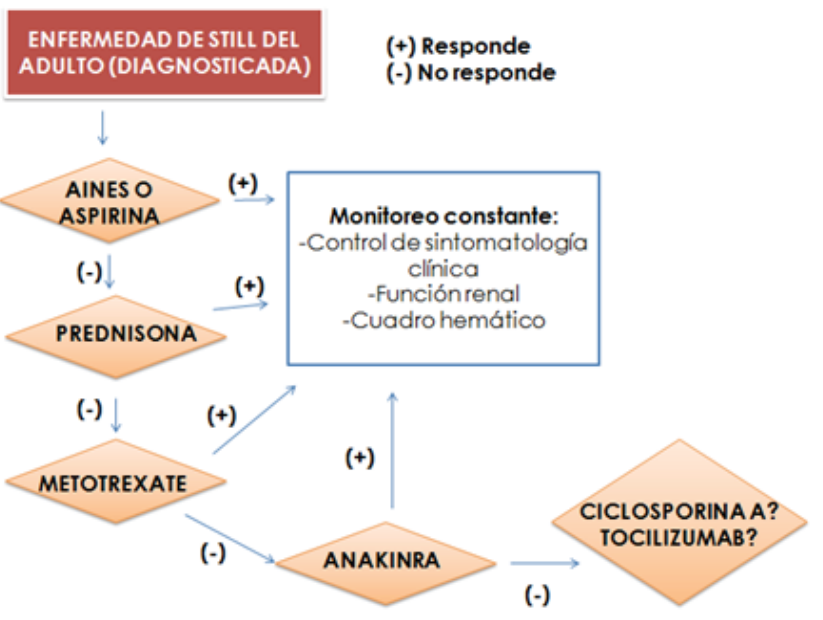

Esquema 1. Algoritmo del tratamiento para la Enfermedad de Still del Adulto.

Criptogénica Organizada con Enfermedad de Still del Adulto es extremadamente rara y por lo general se obtiene adecuada respuesta con el manejo inmunosupresor ${ }^{17}$. El compromiso pulmonar persistente o enfermedad pulmonar restrictiva representan una complicación muy inusual de la enfermedad de Still ${ }^{18}$.

Para el tratamiento de la enfermedad de Still del adulto se ha propuesto el uso de AINES como los medicamentos de primera línea, sin embargo deben ser utilizados en conjunto con esteroides debido a su baja eficacia como monoterapia. El esteroide más utilizado es la Prednisolona como tratamiento de segunda línea y como tercera línea se utiliza Metotrexate seguido de los antagonistas de interleuquina 1 (Anakinra), algunos estudios recomiendan en última instancia los bloqueadores del factor de necrosis tumoral o inhibidores de la interleuquina 6 (Tocilizumab) en casos de refractariedad ${ }^{3,1}$. Medicamento utilizado en el caso en mención posterior a la aplicación del algoritmo de manejo descrito a continuación (Esquema 1).

En cuanto al tratamiento con Tocilizumab (TCZ) en monoterapia se considera eficaz para la enfermedad de Still del adulto puesto que la interleuquina 6 (IL-6) juega un papel crucial en la patogénesis y sus niveles séricos se correlacionan bien con la gravedad. Fitzgerald y cols. propusieron que el factor de necrosis tumoral (TNF- $\alpha$ ) induce la producción de interleuquina 1 (IL-1), que estimula la expresión de IL-6. De hecho, los pacientes que respondieron mal a la Anakinra respondieron adecuadamente al Tocilizumab ${ }^{20}$.
En el presente caso, la terapia con Tocilizumab mejoró rápidamente el compromiso pulmonar y sistémico de la paciente (15 días) con una posología de $8 \mathrm{mg} / \mathrm{kg} /$ dosis. La administración quincenal o mensual de TCZ puede ser necesaria para proteger completamente de la exacerbación a algunos pacientes ${ }^{21}$. Por lo general el tratamiento es bien tolerado, sin embargo, eventos adversos severos como el síndrome de activación de macrófagos debido a un aumento transitorio de los niveles séricos de citoquinas han sido reportados. La gran dificultad en definir la etiología de este síndrome radica en que la enfermedad de Still del adulto por sí misma puede generar síndrome de activación de macrófagos y puede ser difícil de distinguir de los efectos adversos del tratamiento con TCZ y los efectos de la enfermedad ${ }^{22}$.

\section{Conclusión}

La enfermedad de Still del adulto tiene un amplio espectro de presentaciones clínicas, entre éstas y aunque poco común, el compromiso pulmonar; antes de asumir que éste obedece a dicha patología, es importante ser acuciosos en el análisis del cuadro clínico para esclarecer la etiología y descartar infección activa. La gran dificultad que genera este análisis radica en que ambas etiologías generan respuesta inflamatoria sistémica, por lo tanto debemos apoyarnos en otros parámetros clínicos y paraclínicos entre los que resalta los niveles séricos de procalcitonina (negativa en el caso en mención), con la cual podemos decidir el inicio o no de la terapia antibiótica y, en caso de considerar que la afección pulmonar es parte del espectro de la enfermedad de Still, continuar el tratamiento según los algoritmos ya descritos con la intención de lograr un manejo unificado y basado siempre en la mejor evidencia clínica al momento de elegir la terapia más adecuada.

\section{Bibliografía}

1. Bywaters EG. Still's disease in the adult. Annals of the Rheumatic Diseases 1971; 30: 121-133.

2. Still GF. On a Form of Chronic Joint Disease in Children. Med Chir Trans 1897; 80:47.

3. Fautrel B. Adult-onset Still disease. Best Practice \& Research Clinical Rheumatology 2008; 22 (5): 773-792. 
4. Cheema GS, Quismorio FP. Pulmonary involvement in adult-onset Still's disease. Curr Opin Pulm Med. 1999; 5 (5):305-309.

5. Ohta A, Yamaguchi M, Kaneoka H, et al. Adult Still's disease: review of 228 cases from the literature. Journal of Rheumatology 1987; 14: 1139-1146.

6. Aydintug AO, D’Cruz D, Cervera R, Khamashta MA, Hughes GR. Low dose methotrexate treatment in adult Still's disease. J Rheumatol. 1992; 19:431-435.

7. Fautrel B, Le Moël G, Saint-Marcoux B, et al. Diagnostic value of ferritin and glycosylated ferritin in adult onset Still's disease. J Rheumatol. 2001; 28:322.

8. Saghafi M, Sahebari M. Does searching for antineutrophil cytoplasmic antibodies help with the diagnosis of Adult-onset Still's disease? Rheumatol Int. 2013 Mar; 33(3):571-4.

9. Stoica GS, Cohen RI, RossoV LJ. Adult Still's disease and respiratory failure in a 74 year old woman. Postgrad Med J. 2002; 78(916):97-98.

10. Cantor JP, Pitcher WD, Hurd E. Severe restrictive pulmonary defect in a patient with adult-onset Still's disease. Chest 1987; 92(5):939-940.

11. Lioté H, Lioté F, Lenique F, Milleron BJ, Kuntz D, Akoun GM. Adult-onset Still's disease revealed by a pleuropericarditis. Eur Respir J. 1990; 3(9):10641066.

12. Yoshinaga $H$, Koike $R$, Maruyama T, Iizuka $H$, Matsuoka H, Kubota T, Miyasaka N. Still's disease relapse with severe pneumonitis after prolonged remission. Intern Med. 1993; 32(12):902-905.

13. Efthimiou P, Georgy S. Pathogenesis and management of adult-onset Still's disease. Semin Arthritis Rheum. 2006; 36:144-152.

14. Suleiman M, Wolfovitz E, Boulman N, Levy Y. Adult onset Still's disease as a cause of ARDS and acute respiratory failure. Scand J Rheumatol. 2002; 31(3):181-183.

15. Corbett AJ, Zizic TM, Stevens MB. Adult-onset Still's disease with an associated severe restrictive pulmonary defect: a case report. Ann Rheum Dis. 1983; 42(4):452-454.

16. Pouchot J, Sampalis JS, Beaudet F, et al. Adult Still's disease: manifestations, disease Course, and outcome in 62 patients. Medicine 1991; 70:118-136.
17. Sato H, Yokoe I, Nishio S, et al. A case of adult onset Still's disease complicated with cryptogenic organizing pneumonia. Intern Med. 2011; 50(3):247-51.

18. Van Hoeyweghen RJ, De Clerck LS, Van OVel JF, Stevens WJ. Interstitial lung disease and adult-onset Still's disease. Clin Rheumatol. 1993; 12(3):418-421.

19. Puéchal $X$, DeBandt M, Berthelot JM, et al. Tocilizumab in refractory adult Still's disease. Arthritis Care Res. 2011 Jan; 63(1):155-9.

20. de Boysson H, Février J, Nicolle A, Auzary C, Geffray L. Tocilizumab in the treatment of the adult-onset Still's disease: current clinical evidence. Clin Rheumatol. 2013 Jan; 32(1):141-7.

21. Sakai R, Nagasawa H, Nishi E, Okuyama A. Successful treatment of adult-onset Still's disease with tocilizumab monotherapy: two case reports and literature review. Clin Rheumatol. 2012 Mar; 31(3):569-74.

22. Woodrick RS, Ruderman EM. Interleukin 6 inhibition - RA and beyond. Bull NYU Hosp Jt Dis. 2011; 69(3):225-9. 